\title{
The effect of magnocellular-based visual-motor intervention on Chinese children with developmental dyslexia
}

\author{
Yi Qian ${ }^{1,2}$ and Hong-Yan Bi ${ }^{1 *}$ \\ ${ }^{1}$ Key Laboratory of Behavioral Science, Institute of Psychology, Chinese Academy of Sciences, Beijing, China, ${ }^{2}$ University of \\ Chinese Academy of Sciences, Beijing, China
}

Magnocellular (M) deficit theory points out that the core deficit of developmental dyslexia (DD) is the impairment in M pathway, which has been evidenced in many previous studies. Based on the $\mathrm{M}$ deficit, some researchers found that visual intervention focusing on $M$ deficit improved dyslexics' $M$ function as well as reading abilities. However, the number and reliability of these training studies were limited. Therefore, the present study conducted an M-based visual-motor intervention on Chinese children with DD to investigate the relationship between $\mathrm{M}$ deficit and Chinese DD. Intervention programs

Edited by:

Simone Aparecida Capellini, Universidade Estadual Paulista "Júlio de Mesquita Filho", Brazi

Reviewed by:

Maria Luisa Lorusso,

IRCCS "Eugenio Medea", Italy Al Bellamy,

Eastern Michigan University, USA

*Correspondence: Hong-Yan Bi,

Key Laboratory of Behavioral Science, Institute of Psychology, Chinese Academy of Sciences, 16 Lincui Road, Chaoyang District,

Beijing 100101, China bihy@psych.ac.cn

Specialty section: This article was submitted to Educational Psychology, a section of the journa Frontiers in Psychology

Received: 08 July 2015 Accepted: 22 September 2015 Published: 06 October 2015

Citation: Qian Y and Bi H-Y (2015) The effect of magnocellular-based visual-motor intervention on Chinese children with

developmental dyslexia.

Front. Psychol. 6:1529.

doi: 10.3389/fpsyg.2015.01529 included coherent motion detection, visual search, visual tracking, and juggling, which were related to $M$ function. The results showed that $M$ function and phonological awareness of training dyslexic group were improved to a normal level as age-matched normal children after intervention, while non-training dyslexics did not. It supported $\mathrm{M}$ deficit theory, and suggested $\mathrm{M}$ deficit might be the core deficit of Chinese DD.

Keywords: developmental dyslexia, magnocellular pathway, coherent motion detection, visual-motor intervention, Chinese reading

\section{Introduction}

Developmental dyslexia (DD) is a neurobiological reading disorder. Individuals with DD have difficulties in accurate or fluent word recognition, spelling, and word decoding despite adequate instruction and intelligence (Lyon et al., 2003). Although researchers have made efforts in studying on DD, the cause of DD remains controversial. Some researchers pointed out that the cause of DD could be traced back to a more general perceptual dysfunction. Magnocellular (M) deficit theory postulates that the core deficit of DD is the impairment in M pathway (Stein, 2001). M pathway starts from retinal $\mathrm{M}$ ganglion cells in retina, then visual information is conveyed to the M layers of the dorsal lateral geniculate nucleus (LGN) of the thalamus. Then, information was project to the primary visual cortex, and further transferred to the posterior parietal cortex (PPC) via the dorsal stream (also known as the "where" stream), which has been implicated in object localization, motion perception, visual attention, and goal-directed behavior. The dorsal stream includes middle temporal (MT) area, which is known to play a key role in motion perception and is specifically activated when observers are presented with random dot kinematograms (RDKs) containing coherent motion (CM; Goodale and Westwood, 2004; Boden and Giaschi, 2007).

Compared with chronological age-matched (CA) controls, individuals with DD showed less sensitivity in detecting CM (Cornelissen et al., 1995; Talcott et al., 1998, 2003; Witton et al., 1998; Hansen et al., 2001; Conlon et al., 2004; Pellicano and Gibson, 2008). Moreover, CM sensitivity of DD was significantly correlated with pseudoword reading, which reflected the phonological 
processing skills (Talcott et al., 1998; Witton et al., 1998). Prereading children at familial risk of DD exhibited the disability in detecting $\mathrm{CM}$, suggesting deficits in $\mathrm{M}$ pathway occurred before reading commencement (Kevan and Pammer, 2008). A meta-analysis study obtained a relatively large effect size of CM sensitivity in comparison between CA controls and DD, confirming the reliability of CM deficits of DD (Benassi et al., 2010). However, some studies did not support M deficit theory. Ramus et al. (2003) found that only 2 of 16 dyslexic adults had visual $\mathrm{M}$ deficit. Meanwhile, these two visually impaired dyslexics also had auditory and phonological problems, suggesting that visual $\mathrm{M}$ deficit was not an independent core deficit of $\mathrm{DD}$. Sperling et al. (2005) pointed out that deficits in noise exclusion, not $\mathrm{M}$ processing, contributed to the etiology of dyslexia. Additionally, Skottun (2015) pointed out that CM deficit should be attributed to deficits of dorsal stream rather than M pathway; namely, M pathway and dorsal stream should be distinguished. Although $\mathrm{M}$ deficit theory is still debatable, $\mathrm{M}$ deficits have been extensively evidenced in DD.

Some researchers tried to conduct intervention studies on DD. Solan et al. (2004) adopted perceptual accuracy, visual efficiency, guided reading, visual search, and visual scan as training programs. After 15 training sessions, disabled readers' $\mathrm{CM}$ sensitivity, pseudoword reading skills, and reading comprehension were improved (Solan et al., 2004). However, the enhancement of $\mathrm{M}$ function and reading skills might result from the print reading training rather than visual training, because the guided reading program used words as stimuli. Lawton (2011) conducted a direction discrimination task on DD twice a week for 14 weeks, and found that dyslexics enhanced contrast sensitivity (an index of $\mathrm{M}$ function) as well as a mass of reading skills, including reading rates, spelling, word identification and comprehension. The relatively small sample size (only three dyslexics) might limit the reliability of training effects. In a cohort of normal adult readers, either CM or parallel line detection (parvocellular task) training increased the speed of lexical decision, but the correlation between accuracy improvement in lexical decision and improvement of visual function was only found in the CM training group (Chouake et al., 2012). The result suggested that $M$ training seemed to have an advantage over general visual training on reading performance [reaction time (RT) as well as accuracy]. These studies consistently showed that M-based intervention ameliorated reading skills, suggesting the causal role of M pathway in reading development. However, a recent functional magnetic resonance imaging (fMRI) study on dyslexia did not support this opinion. Although dyslexic children showed lower MT activity than CA controls, but similar activity to reading level-matched controls. What's more, the researchers conducted an 8-week phonological-based reading training on DD. They found that dyslexics' MT activity along with reading skills [i.e., real word and pseudoword reading skills and phonological awareness (PA)] were improved, while their performance did not change in the control period (in which a math intervention or no intervention was carried out; Olulade et al., 2013). The result suggested the $M$ deficits attributed to lower reading level and less reading experience rather than the cause of DD.
The above studies were conducted in alphabetic languages. In contrast to the writing systems with specific graphemeto-phoneme correspondence, Chinese is a logographic writing system. Chinese characters have complex visual structures. So, visual processing skills are important for Chinese reading development (Chung et al., 2008; Li et al., 2012; Yang et al., 2013). Previous studies found that Chinese dyslexic children had higher CM thresholds than CA controls (Meng et al., 2011; Qian and $\mathrm{Bi}, 2014$ ), suggesting that Chinese DD had impairments in visual M pathway. An event-related potential (ERP) study showed Chinese DD induced smaller amplitude of visual mismatch negativities (vMMNs) than both age-matched and reading level matched children in the visual $\mathrm{M}$ condition (moving gratings; Wang et al., 2010). A recent intervention study found visual perceptual training significantly decreased the discrimination threshold of visual texture discrimination task (TDT) for Chinese DD. Meanwhile, DD who accepted training exhibited significant and long-lasting enhancement in reading fluency (Meng et al., 2014). However, this study focused on training of general visual perception. Then, how about M pathway function?

Based on previous evidence of $\mathrm{M}$ deficits in Chinese DD (Meng et al., 2011; Qian and Bi, 2014), the present study examined whether M-based visual-motor intervention had a positive effect in $\mathrm{M}$ function and relevant reading-related cognitive skills of DD. Dyslexic children accepted 10 sessions of visual-motor training focusing on $\mathrm{M}$ pathway. Meanwhile, CM detection and reading-related skills were tested on training DD, non-training DD and CA groups. According to previous studies in both alphabetic languages and Chinese, DD exhibited deficits in PA and rapid naming (RAN), which played important roles in reading development (McBride-Chang and Manis, 1996; Wolf and Bowers, 1999; Ho et al., 2000, 2002 ). Moreover, the two skills were closely associated with $\mathrm{M}$ function (Hulslander et al., 2004; Kinsey et al., 2004; Ben-Shachar et al., 2007). Therefore, we adopted PA test and digit RAN test as indexes of children's reading skills.

\section{Materials and Methods}

\section{Participants}

The participants were recruited from ordinary primary schools in Beijing. The study was conducted under the informed consent of their parents, and was approved by the Institutional Review Board of the Institute of Psychology, Chinese Academy of Sciences. All of the participants were right-handed, and had normal hearing and normal or corrected-to-normal vision without ophthalmological or neurological abnormalities. The inclusionary criteria for DD were consistent with previous studies in mainland China (Shu et al., 2006; Wang et al., 2010; Meng et al., 2011; Zhao et al., 2014), including the IQ was above 85 as measured by Combined Raven's Test (Li et al., 1989), meanwhile the written vocabulary test score was at least one and a half standard deviations below corresponding age norm in the Standard Character Recognition Test (Wang and Tao, 1996). Seventeen children with DD and 11 CA children (three female, mean age: 10.42 years, and range: $9-11$ years) took part 
in this study. The children with DD were randomly divided into two groups. One DD group accepted M-based visualmotor intervention (eight children, two females, mean age: 10.63 years, and range: $9-11$ years), while another group did not (nine children, two females, mean age: 10.11 years, and range: 9-11 years). Characteristics of participants were shown in Table 1.

\section{Reading-related Tests PA test}

An oddball paradigm (Bradley and Bryant, 1978) was adopted. Within a trial, three characters were presented orally by the experimenter, and participants were asked to pick out a phonologically odd item among them. There were three types of oddity: onset, rime, and lexical tone. For example, for the three items "tan4", "tong3", and "ji1", "tan4" and"tong3" had the same onset " $\mathrm{t}$ ", which was different from "ji1". Meanwhile, the three items were completely different in rime and lexical tone in order to control for possible confounds. A total of ten trails for each type of oddity was presented. The accuracy of responses was recorded. The test-retest reliability was 0.77 .

\section{RAN Test}

Five digits $(2,4,6,7$, and 9) were used. Digits were repeatedly presented visually in random order on a $6 \times 5$ row-column grid. Participants were asked to name each digit in sequence as quickly as possible. The test was conducted twice, and the scores were averaged as the final score. The total time (s) taken to name all digits was collected and converted to a per-second score. The test-retest reliability was 0.89 .

\section{Magnocelluar Function Test}

We adopted CM detection task to measure magnocelluar pathway function. The experimental paradigm was similar to what used in the study of Solan et al. (2004). Two patches of 300 randomly moving white dots with a speed of $7^{\circ} / \mathrm{s}$ and a lifetime of $225 \mathrm{~ms}$ were presented on the left and right sides of screen with dark background. The luminance of dots was $125 \mathrm{~cd} / \mathrm{m}^{2}$, and the luminance of background was $0.39 \mathrm{~cd} / \mathrm{m}^{2}$, Michelson contrast was $99.4 \%$. The two patches were separated by a horizontal distance subtending $5^{\circ}$, and the size was $10^{\circ}$ wide and $14^{\circ}$ high. The patches of dots were presented for $2300 \mathrm{~ms}$ in each trial. In one patch, all dots moved randomly, while the other patch had a certain percentage of dots moving coherently leftward or rightward. Participants had to judge which patch had such coherently moving dots after patches disappeared. CM threshold was obtained according to a 1-up-1-down staircase procedure; incorrect responses led to an increase in the number of coherent moving dots by $1 \%$, while correct responses led to a decrease by $1 \%$. After 10 reversals, a session was terminated. Threshold was defined by the mean of the number of coherent moving dots of the last six reversals. The experiment included three sessions, and the final CM threshold was the mean of them. The test-retest reliability was 0.74 .

\section{Magnocellular (M)-based Visual-motor Intervention}

The present study adopted M-based visual-motor intervention programs to train dyslexics' $M$ function. One training session included three training projects:

(1) CM detection task. This task aimed to train $M$ function directly. The parameters were similar to that in the above $M$ function test.

(2) Visual search and visual tracking tasks. The tasks focused on eye movement, object localization and visual spatial attention, which were the function of M pathway (Boden and Giaschi, 2007). These tasks were also involved in previous intervention studies (Solan et al., 2004; Lawton, 2011).

(i) In the visual search task, participants were asked to search certain digits (0-9) rapidly and delete them in the sequence from small to large (i.e., $0,1,2,3 \ldots . .9$ ) on a paper of 100 digits. (ii) Visual tracking task included dynamic tracking and static tracking. In dynamic tracking task (12 trials), participants were required to gaze the moving object, pursuit its moving direction, and localize its final position. Static tracking task involved line puzzle (six trials) and maze puzzle (six trials). In the line puzzle task, participants were asked to follow the correct line to find the correct object in connection with the target; in the maze puzzle task, participants were required to find the correct path from entrance to exit. The paradigms of these tasks referred to a previous study (Yu et al., 2006) and the Internet (http://www.eyecanlearn. $\mathrm{com} /$ ). Stimuli in visual search and static visual tracking were presented on papers, while stimuli in dynamic tracking were presented by the computer. The stimuli were different in each training session, and the examples were shown in Figure 1.

(3) Juggling. An fMRI study (Draganski et al., 2004) found that after 3-month juggling training, participants demonstrated a significant transient bilateral expansion in grey matter in MT area. This study suggested that juggling was associated with visual perception and spatial anticipation of moving objects, and was a stronger stimulus for plasticity in the visual areas than in the motor areas (Draganski et al., 2004). Therefore,

TABLE 1 | Characteristics of two developmental dyslexia (DD) groups and one chronological age-matched (CA) group.

\begin{tabular}{|c|c|c|c|c|c|}
\hline & Training DD (1) $(n=8)$ & Non-training DD (2) $(n=9)$ & CA (3) $(n=11)$ & $\boldsymbol{F}$ & Group comparison \\
\hline Age (years) & $10.63(0.52)$ & $10.11(0.33)$ & $10.42(1.07)$ & 1.10 & (1) $=$ (2) $=$ (3) \\
\hline $\mathrm{IQ}$ & $100.13(11.37)$ & 105.63 (10.35) & $109.82(11.91)$ & 1.70 & (1) $=(2)=(3)$ \\
\hline Vocabulary & 2111.02 (306.91) & $2067.30(502.78)$ & $2807.48(349.70)$ & $11.10^{* * *}$ & (1) $=(2)<$ (3) \\
\hline
\end{tabular}

Standard deviations were shown in the parentheses; ${ }^{* * *} p<0.001$. 
A

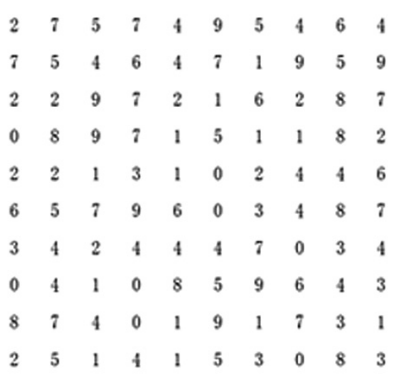

C

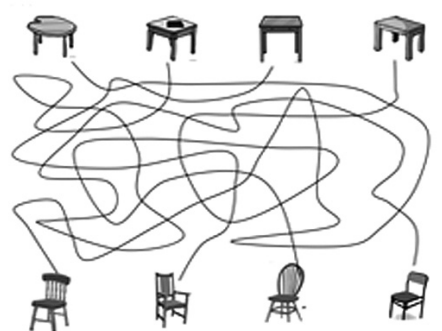

B

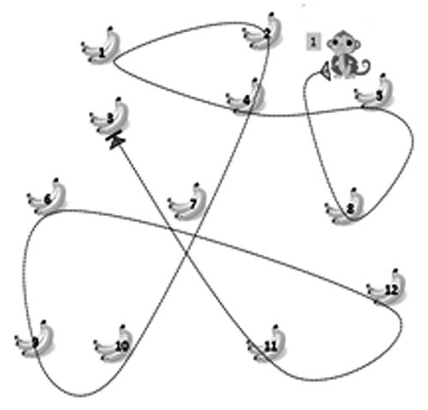

D

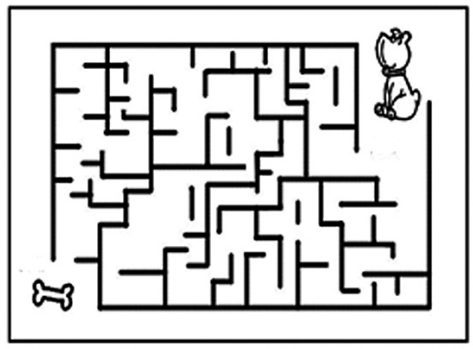

FIGURE 1 | The examples of visual search and visual tracking tasks. (A) The example of visual search; (B) The example of dynamic tracking; (C) The example of line puzzle (a task of static tracking); (D) The example of maze puzzle (a task of static tracking).

juggling was regarded as a M-based intervention program, and adopted to induce the changes of MT area.

\section{Intervention Procedure}

The training DD group accepted ten sessions of M-based visual-motor intervention within 5 weeks with two sessions per week. One session cost about one hour. When training group conducted training programs, non-training dyslexic group, and CA group did free activities. All the three groups accepted reading-related tests and $M$ function test before and after the intervention course.

\section{Results}

CM thresholds, accuracy in PA test, digit RAN speed before and after training for three groups were shown in Table 2. Repeated measures ANOVA were conducted to analyze CM thresholds, accuracy of PA and RAN speed, with group (training DD and non-training DD) as a between-subject factor, and time (pre- and post-) as a within-subject factor. Additionally, we compared the performance difference of the two DD groups and CA group in the three tests, so as to explore dyslexics' deficits before and after training when compared with normal readers.

For CM thresholds, the main effects of group (training DD group and non-training DD group) and time were significant
$[F(1,15)=6.36, p<0.05 ; F(1,15)=11.07, p<0.01]$ the interaction between group and time was also significant $[F(1,15)=7.55, p<0.05]$. Further simple effect analysis showed that there was no significant difference between the two DD groups in the pre-test, while dyslexics in training group had a significantly lower threshold than those in non-training group $(p<0.01)$ in the post-test. For training DD group, post-threshold was significantly lower than pre-threshold $(p<0.01)$, but there was no significant difference for non-training group.

With respect to $\mathrm{PA}$, the main effects of time was significant $[F(1,15)=20.64, p<0.001]$, while the main effects of group (training $\mathrm{DD}$ group and non-training $\mathrm{DD}$ group) was not $[F(1,15)=0.04, p>0.05]$. The interaction between them was significant $[F(1,15)=14.32, p<0.01]$. Simple effect analysis showed that group differences were not significantly different in both pre-PA test and post-PA test The difference of accuracy between pre- and post-test was significant for training DD group (post-accuracy was higher; $p<0.001$ ), but not for non-training DD group.

For digit naming speed, the main effect of time was significant $[F(1,15)=8.23, p<0.05]$, post-naming was faster than prenaming. The main effect of group and the interaction between group and time were not significant.

In order to further explore whether the intervention could improve dyslexics' performance to a normal level, one-way 
TABLE 2 | The performance of pre-test and post-test for three groups.

\begin{tabular}{|c|c|c|c|c|c|c|}
\hline & \multicolumn{3}{|c|}{ Pre- } & \multicolumn{3}{|c|}{ Post- } \\
\hline & Training DD & Non-training DD & CA & Training DD & Non-training DD & CA \\
\hline $\mathrm{CM}$ & 75.88 (26.13) & 83.00 (24.61) & 46.41 (19.59) & 34.19 (11.31) & 79.02 (33.43) & $49.80(30.13)$ \\
\hline PA & $0.47(0.12)$ & $0.53(0.15)$ & $0.72(0.15)$ & $0.63(0.16)$ & $0.54(0.16)$ & $0.71(0.14)$ \\
\hline RAN & $2.66(0.42)$ & $2.69(0.42)$ & $3.19(0.56)$ & $2.87(0.37)$ & $2.85(0.51)$ & $3.50(0.71)$ \\
\hline
\end{tabular}

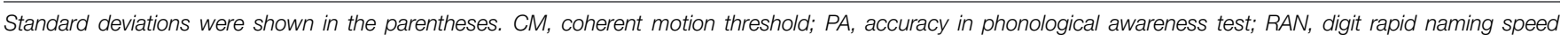
(number per second).

ANOVA was conducted to compare the performance of two DD groups with that of CA group before and after intervention. The results showed that the differences of three groups were significant in pre- and post- CM, PA, and RAN tests; pre-CM: $F(2,25)=7.05, p<0.01$; pre-PA: $F(2,25)=8.20, p<0.01$; preRAN: $F(2,25)=3.88, p<0.05$; post-CM: $F(2,25)=5.92, p<0.01$; post-PA: $F(2,25)=3.17, p=0.06$; and post-RAN: $F(2,25)=4.31$, $p<0.05$. Post hoc analyses indicated that, in the pre-tests, two DD groups had significantly higher CM thresholds $(p s<0.05)$, lower accuracy in PA test $(p s<0.01)$, and slower RAN ( $p s<0.05)$ than CA group, while there was no significant difference between the two DD groups in each of three tests. For post-CM, training DD group and CA group had lower thresholds than non-training group ( $p s<0.05$ ), and there was no difference between training DD group and CA group. For post-PA, only CA group had significantly higher accuracy than non-training group $(p<0.05)$, while there was no significant difference between CA and training groups and between training and non-training groups. For postRAN, CA group had faster naming speed than two DD groups ( $p$ s $<0.05$ ), while there was no significant difference between DD groups.

\section{Discussion}

The present study investigated whether M-based visual-motor intervention could improve $M$ function and reading-related cognitive skills. Compared with age-matched normal children, children with DD exhibited deficits in CM detection, PA and RAN in pre-tests. After intervention, training DD group decreased CM thresholds (i.e., increased CM sensitivity) and increased PA, while non-training DD did not. Additionally, the performance of training DD group in post-CM and PA tests was equal to that of CA group.

Magnocellular-based visual-motor training improved the CM sensitivity of children with DD to a normal level. However, there was no significant difference between pre- and post-CM thresholds for non-training dyslexics. Consistent with previous alphabetic studies (Solan et al., 2004; Lawton, 2011), the present result suggested the $M$ pathway function of dyslexic children benefited from visual intervention. However, the intervention programs included direct CM tasks, then was the enhancement just a practice effect? Actually, dyslexics' deficits in CM were relatively persistent, and not influenced by practice (Conlon et al., 2009), so the enhancement here might not be merely a practice effect, and might benefit from other training tasks.
The current visual-motor intervention involved training focusing on visual search and tracking, which might be related with oculomotor control and visual spatial attention (Hooge and Erkelens, 1999; Kramer et al., 1999; de Brouwer et al., 2002). Juggling has been evidenced induced structural changes in MT area (Draganski et al., 2004). Because of the close association between structure and function (Bullmore and Sporns, 2009), juggling might also exert a positive effect on neural activities in MT areas and further directly influence motion perception. Therefore, dyslexics' $M$ function could be effectively ameliorated by an integrated M-based visual-motor intervention with diverse training programs. However, it was unknown which of these tasks played a dominant role in the improvement of $M$ function, which should be further investigated in the future.

In the post-test, PA of dyslexic children with training was significantly improved, and the accuracy was equivalent to that of CA. However, the accuracy of non-training dyslexics did not differ in the pre- and post-tests, and was still lower than $\mathrm{CA}$, which suggested that $\mathrm{M}$-based visual-motor intervention improved dyslexics' PA, supporting the M deficit theory. Many prior studies have found the association between $M$ function and PA (Talcott et al., 1998; Witton et al., 1998; Hulslander et al., 2004; Kinsey et al., 2004; Ben-Shachar et al., 2007). Another training study found that after a 8-week phonologicalbased reading intervention, MT activity increased along with enhancement of PA (Olulade et al., 2013). It was implied that M deficit was the consequence of impoverished reading rather than the cause of DD. Whereas, Olulade et al. (2013) adopted "seeing stars" as the phonological-based intervention, which addressed visualization of letters, syllables, multisyllables, and words as well as motor/tactile and articulatory aspects of word presentation (Bell, 1997), thereby promoting visual imagery of orthographic presentations as well as PA. So, the intervention program might likewise influence visual system, consequently improving activation in MT area. In contrast, the intervention in the current study was pure perceptual training without the confusion of prints or phonological processing. Thus, it adequately supported $M$ theory, and evidenced that $M$ deficit might be the cause of DD.

Previous studies manifested an association between $M$ function and RAN (Hulslander et al., 2004; Meng et al., 2011; Qian et al., 2015). However, all the three groups in the present study increased RAN speed in the post-test, suggesting the enhancement of RAN might be the outcome of natural development rather than a training effect. The training time was short in the present, which might lead to the lack of training effect on RAN. Therefore, it needed further study to explore whether 
more training sessions would induce larger improvement of naming speed for training dyslexics.

However, the current study had some limitations. Firstly, the relatively small sample size restricted the reliability of the current training effect. Secondly, the non-training groups did free activities, which were not manipulated. Thirdly, the current study lacked character reading or spelling tests, so it was unclear whether the enhancement of reading-related skills could be transferred to general character reading. Finally, all of the dyslexics here had deficits in $M$ pathway. Thus, it remained unknown whether M-based intervention was beneficial to other subtypes of DD. Due to these limitations, caution was needed in drawing conclusions of intervention effect of $M$ pathway. Further studies were needed to confirm the current result by enlarging sample size, manipulating control training programs, adding

\section{References}

Bell, N. (1997). Seeing Stars. San Luis Obispo, CA: Gander Publishing.

Benassi, M., Simonelli, L., Giovagnoli, S., and Bolzani, R. (2010). Coherence motion perception in developmental dyslexia: a meta-analysis of behavioral studies. Dyslexia 16, 341-357. doi: 10.1002/dys.412

Ben-Shachar, M., Dougherty, R. F., Deutsch, G. K., and Wandell, B. A. (2007). Contrast responsivity in $\mathrm{MT}+$ correlates with phonological awareness and reading measures in children. Neuroimage 37, 1396-1406. doi: 10.1016/j.neuroimage.2007.05.060

Boden, C., and Giaschi, D. (2007). M-stream deficits and reading-related visual processes in developmental dyslexia. Psychol. Bull. 133, 346-366. doi: 10.1037/0033-2909.133.2.346

Bradley, L., and Bryant, P. E. (1978). Difficulties in auditory organisation as a possible cause of reading backwardness. Nature 271, 746-747. doi: $10.1038 / 271746 \mathrm{a} 0$

Bullmore, E., and Sporns, O. (2009). Complex brain networks: graph theoretical analysis of structural and functional systems. Nat. Rev. Neurosci. 10, 186-198. doi: $10.1038 / \mathrm{nrn} 2575$

Chouake, T., Levy, T., Javitt, D. C., and Lavidor, M. (2012). Magnocellular training improves visual word recognition. Front. Hum. Neurosci. 6:14. doi: 10.3389/fnhum.2012.00014

Chung, K. K., McBride-Chang, C., Wong, S. W., Cheung, H., Penney, T. B., and Ho, C. S. (2008). The role of visual and auditory temporal processing for Chinese children with developmental dyslexia. Ann. Dyslexia 58, 15-35. doi: 10.1007/s11881-008-0015-4

Conlon, E., Sanders, M., and Zapart, S. (2004). Temporal processing in poor adult readers. Neuropsychologia 42, 142-157. doi: 10.1016/j.neuropsychologia.2003.07.004

Conlon, E. G., Sanders, M. A., and Wright, C. M. (2009). Relationships between global motion and global form processing, practice, cognitive and visual processing in adults with dyslexia or visual discomfort. Neuropsychologia 47, 907-915. doi: 10.1016/j.neuropsychologia.2008.12.037

Cornelissen, P., Richardson, A., Mason, A., Fowler, S., and Stein, J. (1995). Contrast sensitivity and coherent motion detection measured at photopic luminance levels in dyslexics and controls. Vision Res. 35, 1483-1494. doi: 10.1016/00426989(95)98728-R

de Brouwer, S., Yuksel, D., Blohm, G., Missal, M., and Lefèvre, P. (2002). What triggers catch-up saccades during. Vis. Track. 87, 1646-1650.

Draganski, B., Gaser, C., Busch, V., Schuierer, G., Bogdahn, U., and May, A. (2004). Neuroplasticity: changes in grey matter induced by training. Nature 427, 311-312. doi: 10.1038/427311a

Goodale, M. A., and Westwood, D. A. (2004). An evolving view of duplex vision: separate but interacting cortical pathways for perception and action. Curr. Opin. Neurobiol. 14, 203-211. doi: 10.1016/j.conb.2004.03.002

Hansen, P. C., Stein, J. F., Orde, S. R., Winter, J. L., and Talcott, J. B. (2001). Are dyslexics' visual deficits limited to measures of dorsal stream function? Neuroreport 12, 1527-1530. doi: 10.1097/00001756-200105250-00045 general character reading tests, and involving more subtypes of $\mathrm{DD}$, etc.

\section{Summary}

The present preliminary study showed M-based visual-motor training might improve dyslexics' $M$ function and PA, suggesting M-based intervention might be beneficial to Chinese DD.

\section{Acknowledgment}

This research was supported by the grants from Chinese Natural Science Foundation to H-YB (31371044).

Ho, C. S., Chan, D. W., Tsang, S. M., and Lee, S. H. (2002). The cognitive profile and multiple-deficit hypothesis in Chinese developmental dyslexia. Dev. Psychol. 38, 543-553. doi: 10.1037/0012-1649.38.4.543

Ho, C. S.-H., Law, , T. P.-S., and Ng, P. M. (2000). The phonological deficit hypothesis in Chinese developmental dyslexia. Read. Writ. 13, 57-79. doi: 10.1023/A:1008040922662

Hooge, I. T., and Erkelens, C. J. (1999). Peripheral vision and oculomotor control during visual search. Vision Res. 39, 1567-1575. doi: 10.1016/S00426989(98)00213-2

Hulslander, J., Talcott, J., Witton, C., DeFries, J., Pennington, B., Wadsworth, S., et al. (2004). Sensory processing, reading, IQ, and attention. J. Exp. Child Psychol. 88, 274-295. doi: 10.1016/j.jecp.2004.03.006

Kevan, A., and Pammer, K. (2008). Visual deficits in pre-readers at familial risk for dyslexia. Vision Res. 48, 2835-2839. doi: 10.1016/j.visres.2008.09.022

Kinsey, K., Rose, M., Hansen, P., Richardson, A., and Stein, J. (2004). Magnocellular mediated visual-spatial attention and reading ability. Neuroreport 15, 2215-2218. doi: 10.1097/00001756-200410050-00014

Kramer, A. F., Hahn, S., Irwin, D. E., and Theeuwes, J. (1999). Attentional capture and aging: implications for visual search performance and oculomotor control. Psychol. Aging 14, 135-154. doi: 10.1037/0882-7974.14.1.135

Lawton, T. (2011). Improving magnocellular function in the dorsal stream remediates reading deficits. Opt. Vision Dev. 42, 142-154.

Li, D., Chen, G. P., and Jin, Y. (1989). Combined Raven's Matrices Test (CRT): Handbook of Chinese Revised Edition (in Chinese). Shanghai: East China Normal University Press.

Li, H., Shu, H., McBride-Chang, C., Liu, H., and Peng, H. (2012). Chinese children's character recognition: visuo-orthographic, phonological processing and morphological skills. J. Res. Read. 35, 287-307. doi: 10.1111/j.14679817.2010.01460.x

Lyon, G., Reid Shaywitz, S. E., and Shaywitz, B. A. (2003). A definition of dyslexia. Ann. Dyslexia 53, 1-14. doi: 10.1007/s11881-003-0001-9

McBride-Chang, C., and Manis, F. R. (1996). Structural invariance in the associations of naming speed, phonological awareness, and verbal reasoning in good and poor readers: a test of the double deficit hypothesis. Read. Writ. 8, 323-339. doi: 10.1007/BF00395112

Meng, X., Cheng-Lai, A., Zeng, B., Stein, J. F., and Zhou, X. (2011). Dynamic visual perception and reading development in Chinese school children. Ann. Dyslexia 61, 161-176. doi: 10.1007/s11881-010-0049-2

Meng, X., Lin, O., Wang, F., Jiang, Y., and Song, Y. (2014). Reading performance is enhanced by visual texture discrimination training in chinesespeaking children with developmental dyslexia. PLOS ONE 9:e108274. doi: 10.1371/journal.pone.0108274

Olulade, O. A., Napoliello, E. M., and Eden, G. F. (2013). Abnormal visual motion processing is not a cause of dyslexia. Neuron 79, 180-190. doi: 10.1016/j.neuron.2013.05.002

Pellicano, E., and Gibson, L. Y. (2008). Investigating the functional integrity of the dorsal visual pathway in autism and dyslexia. Neuropsychologia 46, 2593-2596. doi: 10.1016/j.neuropsychologia.2008.04.008 
Qian, Y., and Bi, H. Y. (2014). The visual magnocellular deficit in Chinesespeaking children with developmental dyslexia. Front. Psychol. 5:692. doi: 10.3389/fpsyg.2014.00692

Qian, Y., Deng, Y., Zhao, J., and Bi, H. Y. (2015). Magnocellular-dorsal pathway function is associated with orthographic but not phonological skill: fMRI evidence from skilled Chinese readers. Neuropsychologia 71, 84-90. doi: 10.1016/j.neuropsychologia.2015.03.024

Ramus, F., Rosen, S., Dakin, S. C., Day, B. L., Castellote, J. M., White, S., et al. (2003). Theories of developmental dyslexia: insights from a multiple case study of dyslexic adults. Brain 126, 841-865. doi: 10.1093/brain/awg076

Shu, H., McBride-Chang, C., Wu, S., and Liu, H. (2006). Understanding chinese developmental dyslexia: morphological awareness as a core cognitive construct. J. Educ. Psychol. 98, 122-133. doi: 10.1037/0022-0663.98.1.122

Skottun, B. C. (2015). ). The need to differentiate the magnocellular system from the dorsal stream in connection with dyslexia. Brain Cogn. 95, 62-66. doi: 10.1016/j.bandc.2015.01.001

Solan, H. A., Shelley-Tremblay, J., Hansen, P. C., Silverman, M. E., Larson, S., and Ficarra, A. (2004). M-Cell deficit and reading disability: a preliminary study of the effects of temporal vision-processing therapy. Optometry 75, 640-650. doi: 10.1016/S1529-1839(04)70211-0

Sperling, A. J., Lu, Z. L., Manis, F. R., and Seidenberg, M. S. (2005). Deficits in perceptual noise exclusion in developmental dyslexia. Nat. Neurosci. 8, 862-863. doi: $10.1038 / \mathrm{nn} 1474$

Stein, J. (2001). The magnocellular theory of developmental dyslexia. Dyslexia 7 , 12-36. doi: 10.1002/dys.186

Talcott, J. B., Gram, A., Van Ingelghem, M., Witton, C., Stein, J. F., and Toennessen, F. E. (2003). Impaired sensitivity to dynamic stimuli in poor readers of a regular orthography. Brain Lang. 87, 259-266. doi: 10.1016/s0093-934x(03)00105-6

Talcott, J. B., Hansen, P. C., Willis-Owen, C., McKinnell, I. W., Richardson, A. J., and Stein, J. F. (1998). Visual magnocellular impairment in adult developmental dyslexics. Neuro Ophthalmol. 20, 187-201. doi: 10.1076/noph.20.4.187.3931

Wang, J. J., Bi, H.-Y., Gao, L.-Q., and Wydell, T. N. (2010). The visual magnocellular pathway in Chinese-speaking children with developmental dyslexia. Neuropsychologia 48, 3627-3633. doi: 10.1016/j.neuropsychologia.2010.08.015

Wang, X. L., and Tao, B. P. (1996). Chinese Character Recognition Test Battery and Assessment Scale for Primary School Children. Shanghai: Shanghai Education Press.

Witton, C., Talcott, J. B., Hansen, P. C., Richardson, A. J., Griffiths, T. D., Rees, A., et al. (1998). Sensitivity to dynamic auditory and visual stimuli predicts nonword reading ability in both dyslexic and normal readers. Curr. Biol. 8, 791-797. doi: 10.1016/S0960-9822(98)70320-3

Wolf, M., and Bowers, P. G. (1999). The double-deficit hypothesis for the developmental dyslexias. J. Educ. Psychol. 91, 415-438. doi: 10.1037/00220663.91.3.415

Yang, Y-L., Guo, J.-P., Richman, L. C., Schmidt, F. L., Gerken, K. C., and Ding, Y. (2013). Visual skills and chinese reading acquisition: a meta-analysis of correlation evidence. Educ. Psychol. Rev. 25, 115-143. doi: 10.1007/s10648013-9217-3

Yu, X. D., Lu, F., and Jiang, L. F. (2006). Vision training and its effects on reading speed in low vision children. Chin. J. Opt. Ophthalmol. 8, 325-327.

Zhao, J., Qian, Y., Bi, H. Y., and Coltheart, M. (2014). The visual magnocellulardorsal dysfunction in Chinese children with developmental dyslexia impedes Chinese character recognition. Sci. Rep. 4, 7068. doi: 10.1038/srep 07068

Conflict of Interest Statement: The authors declare that the research was conducted in the absence of any commercial or financial relationships that could be construed as a potential conflict of interest.

Copyright (c) 2015 Qian and Bi. This is an open-access article distributed under the terms of the Creative Commons Attribution License (CC BY). The use, distribution or reproduction in other forums is permitted, provided the original author(s) or licensor are credited and that the original publication in this journal is cited, in accordance with accepted academic practice. No use, distribution or reproduction is permitted which does not comply with these terms. 